\title{
Localization and Habituation of Sensory Evoked DC Responses in Cat Cortex
}

\author{
Marvin E. Lickey and Stephen S. Fox ${ }^{1}$ \\ Department of Psychology, University of Michigan, Ann Arbor, Michigan
}

Received January 31, 1966

\begin{abstract}
Using calomel electrodes and chopper stabilized amplifiers, sensory evoked d-c responses at the cortical surface were recorded from the primary visual, secondary visual, auditory and somatic areas of the left hemisphere in thirty acutely prepared immobilized cats. The stimuli were light from an incandescent bulb, a hissing sound and a mild shock. Responses were quantified on line by automatic computation of area under the response and by waveform averaging. All four cortical loci could respond to all three stimuli, but by an algebraic analysis of response amplitudes the responses could be fractionated into two components, one of which was localized, the other diffuse. The local component is stimulus bound and is distributed such that the response of the classical sensory area relevant to the stimulus is negative to the response of the other sensory areas, regardless of the over-all response polarity. The diffuse component is, on the average, negative in polarity and has a longer latency and duration than the local component. It occurs primarily as an aftereffect of stimulation and is more readily evoked by shock and hiss than by light. The diffuse component and the local component are, therefore, distinguished from one another both by their localization and their time course. Both the diffuse component and the local component showed considerable habituation during a 1.5 to 2.0 hour long series of fifty stimulations. The responses probably reflect the joint action of both specific and diffuse cortical inputs, but it is unlikely that they influence the production of action potentials in cortical neurons.
\end{abstract}

\section{Introduction}

Following the suggestion of Gerard (5) that d-c field potentials may have an important synchronizing influence on cortical neurons, numerous

1 This research was a part of a dissertation submitted by the senior author in partial fulfillment of the requirements for the Doctor of Philosophy degree in the University of Michigan. Special thanks are extended to Drs. James Olds, James B. Ranck and E. L. Walker for their constructive advice and criticism. The research was supported by NSF grant G21446, GB1711, and NIH grant Mh-11834-01 to Dr. Fox, and the experiments were conducted in the facilities of the Mental Health Research Institute. Dr. Lickey's present address is Division of Biology, California Institute of Technology, Pasadena, California. That of Dr. Fox is Department of Psychology, University of Iowa, Iowa City, Iowa. 
papers have been published describing sensory evoked slow potential changes in the mammalian cortex. These papers may be categorized roughly into two groups. One group has placed emphasis on the relation of the cortical steady potential (SP) $)^{2}$ to cortical activation or behavioral arousal $(2,3,9,26,27)$. A second group, to which the present paper belongs, has explored the relation of cortical slow potentials to specific sensory and perceptual activities. Libet and Kahn (17) reported that sensory stimulation evokes surface negative $\mathrm{d}-\mathrm{c}$ responses of 0.2 to $0.3 \mathrm{mv}$ in the appropriate cortical sensory areas, and in a pioneering series of papers, Köhler and his colleagues (12-15) proposed that sensory evoked $\mathrm{d}-\mathrm{c}$ responses might play an important role in the determination of perception. Slow potential changes have also been described in response to stimulation of sensory nerves and the thalamic relay nuclei $(2,6-9)$.

The present study describes the cortical distribution and persistence of $\mathrm{d}-\mathrm{c}$ responses evoked by receptor stimulation in three modalities. We hope that the findings will contribute to the evaluation of $d-c$ responses as useful correlates of perception and attention.

In one of the few other papers on this subject, Gumnit (11) reported that $d-c$ responses to auditory stimulation may be discretely localized within the auditory cortex and that the responses are subject to unusually rapid habituation. The data reported in the present paper corroborate Gumnit's findings and extend them to the visual and somatic modalities. On-line quantification of response magnitude, duration and polarity has allowed for statistical analyses which can separate reliable response properties from a rather broad band of individual variation.

\section{Methods}

The data were collected from thirty acutely prepared immobilized and procainized cats weighing 2.5 to $4.0 \mathrm{~kg}$.

Surgery. Under ether anesthesia the following procedures, lasting 40 to 60 min, were carried out: (i) placement of an endotracheal tube; (ii) cannulation of the radial vein; (iii) placement in a David Kopf stereotaxic instrument; $(i v)$ insertion of two 27-gauge, 0.5 -in. hypodermic needles into the right front foot pad for subsequent electrical stimulation; $(v)$ insertion of a 22-gauge, 1-in. hypodermic needle into the right rear thigh for monitoring EKG; (vi) application of atropine to the eyes to prevent the

"According to the convention of O'Leary and Goldring (21), "SP" is the absolute value of the maintained cortical potential with respect to some neutral reference. "D-C responses" are evoked transient changes in SP. 
pupillary reflex; and (vii) exposure of the dura mater over four sensory areas of the left hemisphere for the purpose of recording. Circular cranial openings 3 to $6 \mathrm{~mm}$ in diameter were made with a dental burr. They were located visually according to cranial landmarks so as to expose the primary visual $\left(V_{1}\right)$, secondary visual $\left(V_{2}\right)$, somatic $(S)$ and auditory $(A)$ areas. No attempt was made to record from precisely homologous points in different preparations, and occasionally a placement fell slightly outside the target area. In the figures and text which follow, the actual recording sites assume the name of the intended target areas. In a few preparations the dura mater was removed. This procedure was discontinued, however, since it risked cortical damage, and the responses recorded from dura were more reliable but only slightly smaller than those recorded from pia mater.

The drug d-tubocurarine chloride (Upjohn) was then injected through the radial vein cannula in a dose sufficient to block spontaneous respiration, and the animal was maintained with a Harvard respiration pump. Procaine was applied intradermally at all surgical wounds and stereotaxic pressure points. Additional curare was injected when necesasry to maintain complete paralysis. At least 3 hours were allowed for ether dissipation before experimentation began. General anesthesia was contraindicated by reports that $d-c$ responses are suppressed by anesthesia, especially the barbiturates $(1,14)$. These reports were corroborated in our own laboratory. The present experiments involved no strong stimulation, and EEG rhythms typical of drowsiness and sleep were often observed in the immobilized animal.

Electrodes. Calomel electrodes (Beckman 40463) were led to the dural surface at each of the four recording sites via latex side arms filled with saturated $\mathrm{KCl}$ solution. Contact with the dura mater was made through a saline-agar gel bridge confined in a glass pipette having a pore diameter of 1 to $1.5 \mathrm{~mm}$. The reference electrode, also a calomel half cell, was placed on the occipital protuberance from which all tendons and periosteum had been removed. In three preparations the secondary visual placement was sacrificed in favor of an auxiliary reference electrode (pipettc, o.d. = $1.5 \mathrm{~mm}$ ) led from the left lateral ventricle. Responses recorded against this reference were substantially the same in both amplitude and polarity as those recorded against the bone reference. All the pipettes were sealed in place with either very soft paraffin $(\mathrm{mp}=40.5 \mathrm{C})$ or agar gel. Once sealed, the pipettes were not disturbed. Electrode potential drift was less than $50 \mu \mathrm{v} / \mathrm{hr}$ when a pair of electrodes was shorted through a saline bridge. In addition to the recording pipettes, a thermistor housed in the tip of a 21 - 
gauge hypodermic needle was inserted 1 to $2 \mathrm{~mm}$ into the right primary visual cortex.

Maintenance of the Preparation. The EKG, EEG, cortical temperature, $\mathrm{CO}_{2}$ in respired air, and the cortical steady potential (SP) were continuously monitored throughout the experiment. Long-term stability of all these parameters was, of course, desired. The EKG was quite stable in both waveform and rate with typical values for heart rate falling between 180 and 200 per min. Occasionally cardiac acceleration accompanied an injection of d-tubocurarine. The EEG showed the normal mix of frequencies typical of an aroused animal with occasional intrusions of sleep patterns. One preparation developed seizure activity due to respiratory failure, and the resulting data were rejected from the analysis, reducing the total number of preparations to twenty-nine. After 4 to 5 hours of experimentation there sometimes appeared high-amplitude slow waves which could not be blocked by stimulation. Cortical temperature was maintained at $37.5 \pm 0.5 \mathrm{C}$ by adjusting the output of an electrostatically shielded heat lamp mounted about 2 feet above the preparation. The percentage of $\mathrm{CO}_{2}$ in respired air was monitored on a Harvard model 2000 $\mathrm{CO}_{2}$ analyzer. The system used did not provide an undiluted sample of respired air, and it was therefore impossible to obtain an accurate value for percentage of $\mathrm{CO}_{2}$. Empirical trials on pilot animals, however, established a stable $\mathrm{CO}_{2}$ reading $(1.9-2.2 \%)$ associated with a healthy preparation, and the pumping rate of the respirator was adjusted to maintain the $\mathrm{CO}_{2}$ at this level. The cortical SP varied in different preparations between +2 and $+8 \mathrm{mv}$. It often started to drift negatively toward zero after 4 to 5 hours of experimentation.

The data reported here were collected during the initial 1.5 to 2 hours of experimentation, before any signs of systemic instability had appeared. If transient instabilities appeared, for instance in heart rate or SP due to an injection of d-tubocurarine, the experimental procedures were interrupted until stability was recovered.

Stimuli. The experiments were conducted in a dimly lit room with background equipment noise of about $50 \mathrm{db}$. A visual, auditory or somatic stimulus of 10 -sec duration was presented automatically at a mean rate of $1 / 120 \mathrm{sec}$ for a total of fifty trials. The interval between the onsets of any two trials varied in a random order between 45 and 195 sec in twenty-one equal steps of $7.5 \mathrm{sec}$. The visual stimulus was a $100-\mathrm{w}$ incandescent lamp 5 feet in front of the cat. The auditory stimulus was a hissing sound gen- 
erated by a solenoid controlled air jet. The sound intensity at the cat's head was $80 \mathrm{db}$ referred to 0.0002 dyne $/ \mathrm{cm}^{2}$. The somatic stimulus was a mild shock consisting of a train of square pulses delivered to the right front foot pad. The shock parameters were: frequency $=50 / \mathrm{sec}$; duration $=0.3 \mathrm{msec}$; amplitude $=5 \mathrm{v}$. The threshold voltage for evoking a $\mathrm{d}-\mathrm{c}$ response was typically 2 to $3 \mathrm{v}$.

Analysis of Data. The d-c responses and EEG were recorded on a chopper stabilized eight-channel polygraph. The final stage output of any channel could be fed to one or all of three other devices: an oscilloscope for examination of evoked potentials and EEG components; a TMC CAT $400 \mathrm{~B}$ computer for waveform averaging; and a ten-channel Sterling LM-10 electronic analog computer for on-line computation of area under the response. The logic of this integration procedure is given wtih Fig. 1.

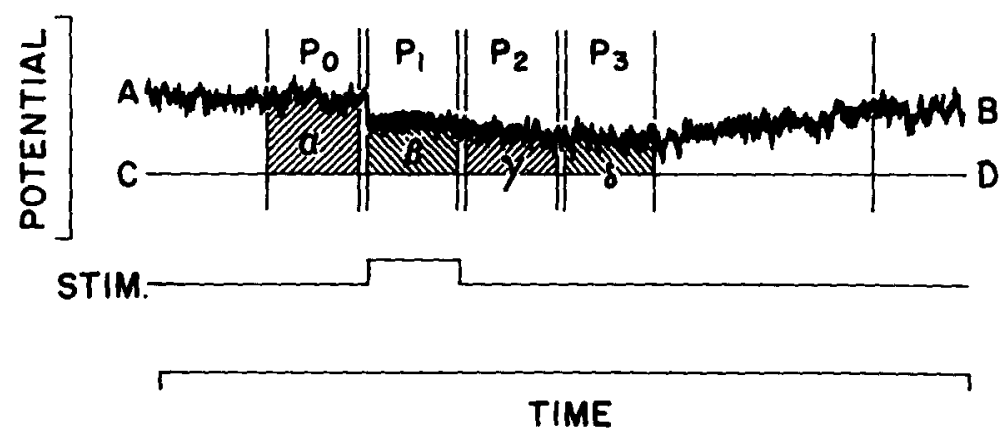

FiG. 1. Computation of area under response. Tracing $A B$ is the potential recorded from brain. Line $C D$ is an arbitrary reference potential. The $d-c$ response to be analyzed is broken into three equal integration periods, $P_{1}, P_{2}$, and $P_{3}$. Stimulation occurs during $P_{1}, P_{0}$ is an equal prestimulation integration period which is used to establish a baseline. The area between $A B$ and $C D$ is computed separately for $P_{0}, P_{1}$, $\mathrm{P}_{2}$, and $\mathrm{P}_{3}$ yielding areas $\alpha, \beta, \gamma$, and $\delta$, respectively. The response magnitude in $\mu \mathrm{v}-$ sec is then given by the quantities $(\beta-\alpha),(\gamma-\alpha)$, and $(\delta-\alpha)$.

Verification of Electrode Placement. At the end of an experiment the animal was killed with sodium pentobarbital, and the pipettes were withdrawn leaving behind tracks through the agar or parafin seal. Pins were then inserted into the brain along the axis of the pipette tracks, the skull was removed, and the position of the pins on the cortical surface was photographed. 


\section{Results}

Direct-current responses of varying amplitude and reliability were evoked in all preparations. Figure 2 summarizes the most commonly observed variations with representative examples selected from four preparations. The responses ranged from 20 to $400 \mu \mathrm{v}$ in peak amplitude and from 3 to $60 \mathrm{sec}$ in duration. Biphasic waves and responses to stimulus offset were sometimes observed (Fig. 2A). Response components which outlasted

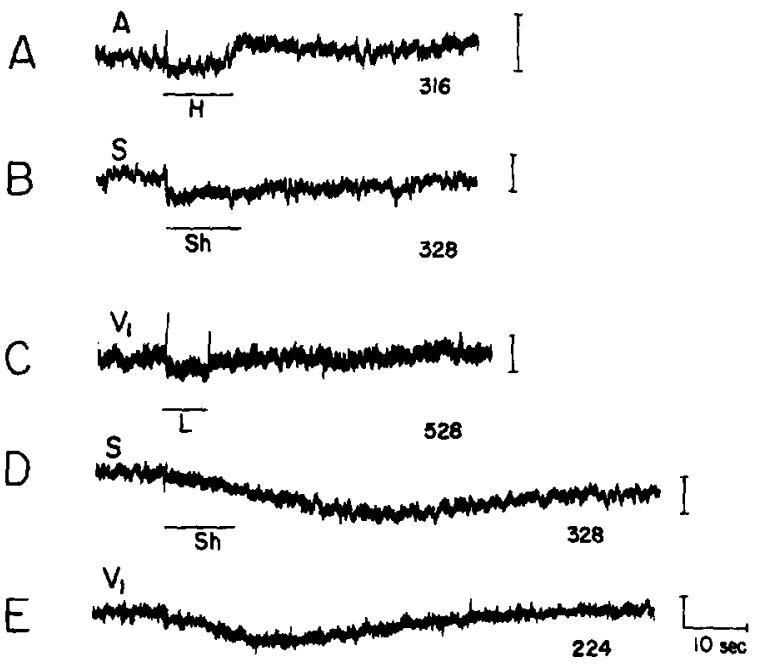

FIG. 2. Summary of $d-c$ responses. $A$ : auditory area. $S$ : somatic area. $V_{1}$ : primary visual area. Gain calibration: $250 \mu \mathrm{v}$. H: hiss stimulus. L: light stimulus. Sh: shock stimulus. Stimulus duration: $10 \mathrm{sec}$. Preparation identification number at lower right of each trace. Positive up.

stimulation were less reliable than the initial deflection occurring during the period of stimulation.

Two characteristic wave shapes were distinguishable among many variations. Responses of one type rise suddenly to a peak within 1 or $2 \mathrm{sec}$, are of short duration, and may be accompanied by evoked potentials at the onset or offset of stimulation, or both (Fig. 2A, B, C). Responses of the second type approach their peak slowly over 5 to $30 \mathrm{sec}$ and have greater amplitude and duration than the fast rising responses (Fig. 2D). Occasionally the two wave shapes appear to be superimposed. In about onefourth of the preparations there were spontaneous fluctuations in SP which resembled the larger slowly rising evoked responses (Fig. 2E). In about 
half these preparations stimuli sometimes appeared to trigger responses similar to the spontaneous fluctuations.

Localization. Figure 3 shows the responses of a single preparation to all three of the stimuli within a 3 -min interval. Light (left) evokes a negative



FIG. 3. Responses of a single preparation to three stimuli. $\mathrm{V}_{1}$ : primary visual area. $\mathrm{V}_{2}$ : secondary visual area. $\mathrm{S}$ : somatic area. $\mathrm{A}$ : auditory area. $\mathrm{L}$ : light stimulus. $\mathrm{H}$ : hiss stimulus. Sh: shock stimulus. Recording sites shown in brain drawing. Positive up.

response in the two visual leads $\left(V_{1}\right.$ and $\left.V_{2}\right)$; hiss (middle) evokes a negative response in the auditory lead (A); shock (right) evokes a negative response in the somatic lead (S). Yet the responses are not wholly confined to the primary area relevant to the stimulated modality. Light also evokes a positive response in the autditory area, hiss a weaker negative response in the visual areas, and shock a small initially positive response in the auditory area. In every case, however, the response of the stimulus relevant cortical area is negative to the responses of the other three areas.

This rule of localization is again demonstrated in Fig. 4 which shows CAT averaged responses to eight shock stimulations in three preparations. In Fig. 4A the somatic area shows the smallest response in a preparation responding positively in all leads. In $\mathbf{B}$ the somatic area shows the largest response in a preparation responding negatively in all leads. In $\mathrm{C}$ the somatic area responds negatively while other loci respond positively. Response distributions of the type shown in B were smaller in amplitude and less frequently observed than those of the type shown in A and C. In C it may be noted that the relative negativity of the somatic area holds only 
during the period of stimulation; the poststimulation response components are polarity reversed with the somatic area being the most positive.

In general, the response of the coritcal area relevant to the stimulus is negative with respect to the other cortical areas during the period of

A

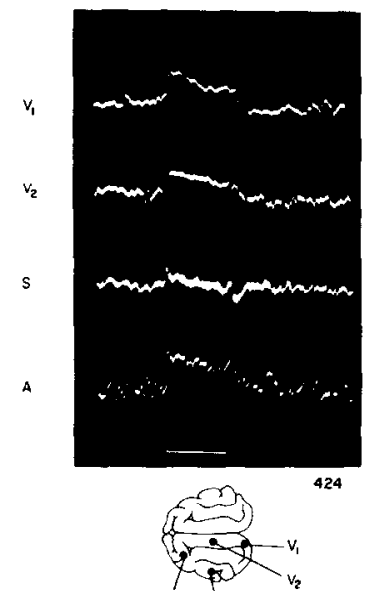

B
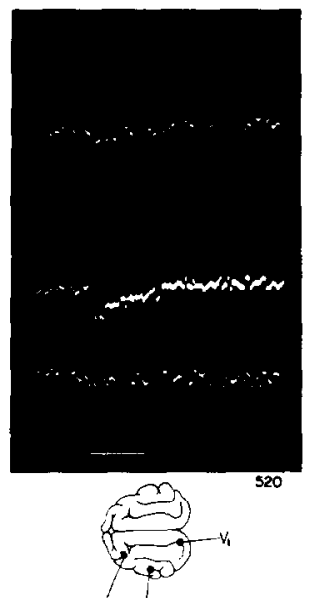

C



Fic. 4. Response to shock in three preparations as shown by CAT 400 B. $V_{1}$ : primary visual area. $\mathrm{V}_{\mathrm{z}}$ : secondary visual area. $\mathrm{S}$ : somatic area. A: auditory area. Recording sites shown on brain drawings. In B three loci recorded using ventricle reference. Stimulus duration: $10 \mathrm{sec}$. Analysis time: $42 \mathrm{sec}$. Vertical axis uncalibrated. Each tracing based on eight responses. Positive up.

stimulation. For ease of reference this rule of localization will henceforth be called the primary negative rule.

Figure 5 shows the consistency of the primary negative rule across preparations. The recording site of the stimulus relevant lead is indicated for all but one of the twenty-nine preparations. (There was no record of electrode placement for one of the eight preparations receiving light.) If the stimulus-relevant area obeyed the primary negative rule, the recording site is indicated as a "plus"; if it failed to obey the rule, the recording site is indicated as a "circle." (The eighth preparation receiving light would be represented as a plus in the vicinity of the primary visual area.)

The primary negative rule held without qualification for twenty-two of the twenty-nine preparations, seven of the eight receiving light, nine of the twelve receiving shock and six of the nine receiving hiss. For light and shock all the exceptions were recorded from cortical sites slightly outside 


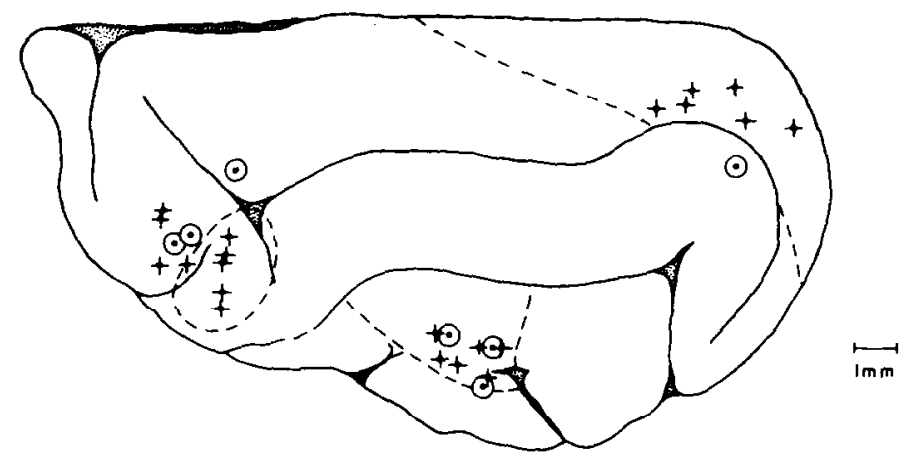

FIG. 5. Consistency of primary negative rule. The site of the stimulus relevant recording pipette for each preparation is shown as a " + " if the response obeyed the primary negative rule, as a " $\odot$ " if it violated the rule. The response is defined as the mean response integral for the first twenty-five trials. The three primary areas are enclosed by dashed lines.

the primary area, and it is therefore perhaps fair to conclude that there are no exceptions at all in these two modalities. For hiss, however, the exceptions appear to be genuine.

Ilabituation. Figure 6 shows the habituation of the response to shock in a single preparation with six trials selected at ten trial intervals from the fifty trial series. The largest responses were nearly always observed during the first ten to fifteen trials of the series, falling to about half their original amplitude after twenty-five to thirty trials. At the end of the fifty trial series, dishabituation due to the intrusion of novel stimuli was seldom observed, but after withholding all stimulation for as long as $45 \mathrm{~min}$, habituated responses could recover or even exceed their original size.

The course of habituation in all preparations was analyzed quantitatively by the use of response integrals as an index of response magnitude (Fig. 1), and the results of the analysis are presented in Figs. 7-10.

The construction of these figures was suggested by the finding, described above, that the response of the cortical area relevant to the stimulus is negative to other sensory areas. This local component is presented independently of the total response according to the following albegraic procedure: The nonlocalized portion of a response, that is to say the diffuse component, was defined as the mean response of all four loci. Four local components were then obtained by computing the algebraic deviation of the response at each locus from the diffuse component. After the diffuse and local components had been computed for each preparation, all the data 
within each stimulation group were pooled and means were computed for blocks of five trials. To eliminate distortion of the group means by preparations giving unusually large responses, the response magnitudes were first rendered normal by converting to percentage of the mean absolute value

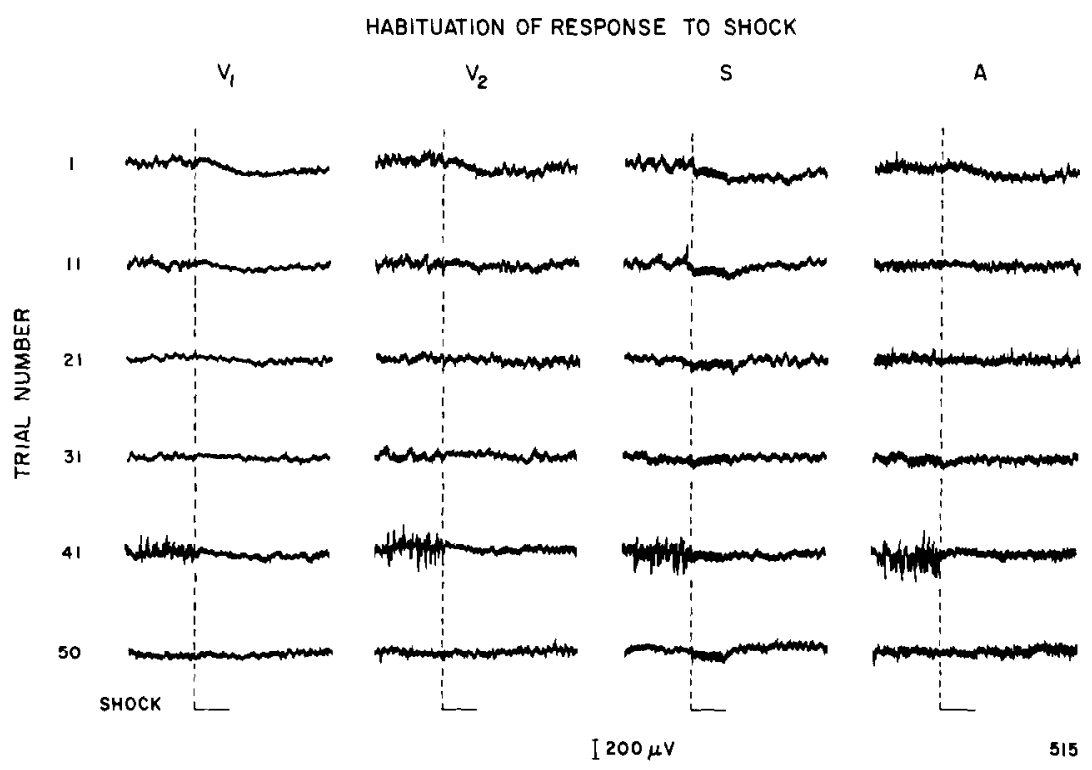

Fic. 6. Habituation of response to shock. $V_{1}$ : primary visual area. $V_{2}$ : secondary visual area. S: somatic area. A: auditory area. Onset of shock stimulus indicated by vertical dashed lines. Stimulus duration: $10 \mathrm{sec}$. Positive up.

of the response integral during the period of stimulation $\left(P_{1}\right)$ averaged across all fifty trials and across all loci. Thus, in Figs. 7-9 a local component of $-100 \%$ indicates an average negative deviation of the same absolute value as the average raw response during stimulation. The baseline is the mean of all four areas, and the degree of separation between the curves represents the degree to which the responses of the different sensory areas are different from one another. In Fig. 10, a diffuse component of $-100 \%$ indicates that the mean of all four loci is negative and has the same absolute value as the average raw response during stimulation.

Local Component. In the curves for the period of stimulation $\left(\mathrm{P}_{1}\right)$, the primary negative rule is expressed by the negativity of the local component in the stimulus relevant receiving areas (Figs. 7-9). The local components for the nonrelevant areas are either very small or positive in polarity. With 
repetition the responses of the various areas becomes more nearly equivalent.

In the curves for the poststimulation periods $\left(\mathrm{P}_{2 .}\right.$ and $\mathrm{P}_{3}$ ), there is no evidence for any orderly change with repetition. The local components are greatly reduced, and with the exception of shock during $P_{: .}$, the curves appear to be randomly intermingled. On this basis it may be concluded that the primary negative rule applies chiefly to the initial portion of the

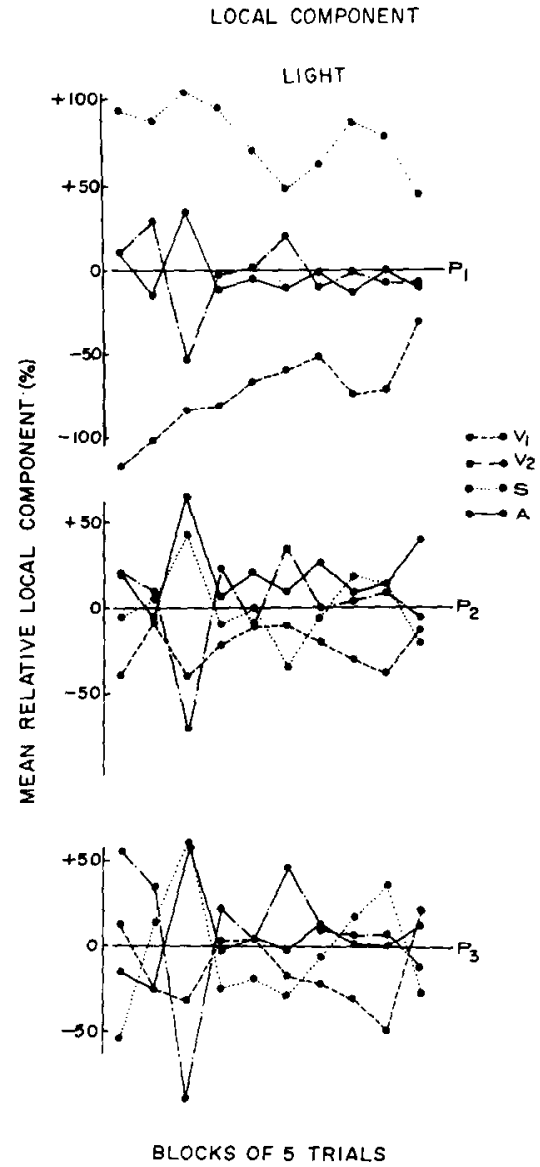

FIG. 7. Habituation of mean local component to light. $\mathrm{N}=8 . \mathrm{P}_{1}$ : integration period one, period of stimulus. $P_{2}$ : integration period two, first poststimulus period. $P_{3}$ : integration period three, second poststimulus period. $V_{1}$ : primary visual area. $V_{2}$ : secondary visual area. S: somatic area. A: auditory area. For definition of mean relative local component, see text. 
response occurring during the period of stimulation, and that response localization is reduced during the course of habituation.

Diffuse Component. Several properties of the diffuse component distinguish it from the local component, as can be seen in Fig. 10. The diffuse component is generally negative in polarity, and it is larger for hiss and shock than for light. During the poststimulation periods $\left(\mathrm{P}_{2}\right.$ and $\left.\mathrm{P}_{3}\right)$ it is larger than during the period of stimulation $\left(P_{1}\right)$. When it is large at the

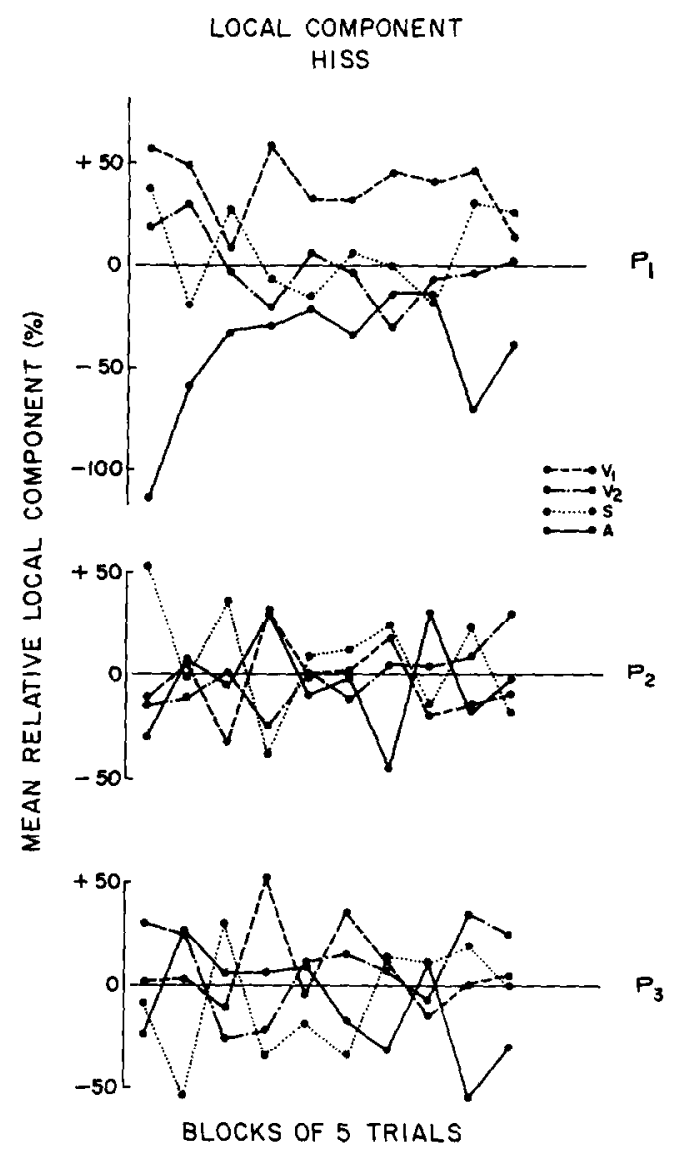

Fic. 8. Habituation of mean local component to hiss. $N=9$. $\mathbf{P}_{1}$ : integration period one, period of stimulus. $\mathrm{P}_{2}$ : integration period two, first poststimulus period. $P_{3}$ : integration period three, second poststimulus period. $V_{1}$ : primary visual area. $\mathrm{V}_{2}$ : secondary visual area. $\mathrm{S}$ : somatic area. $\mathrm{A}$ : auditory area. For definition of mean relative local component, see text. 
beginning of the fifty trial series, as with hiss and shock, there is a significant reduction with repetition. In the case of light, however, the course of habituation appears to be nonmonotonic, having a positive peak at trials sixteen through twenty. Examination of the curves for individual animals indicated that five of the eight preparations receiving light gave a large positive peak in this region. It will also be noted that the diffuse component to light shows no over-all decrement with repetition, and during $\mathrm{P}_{1}$

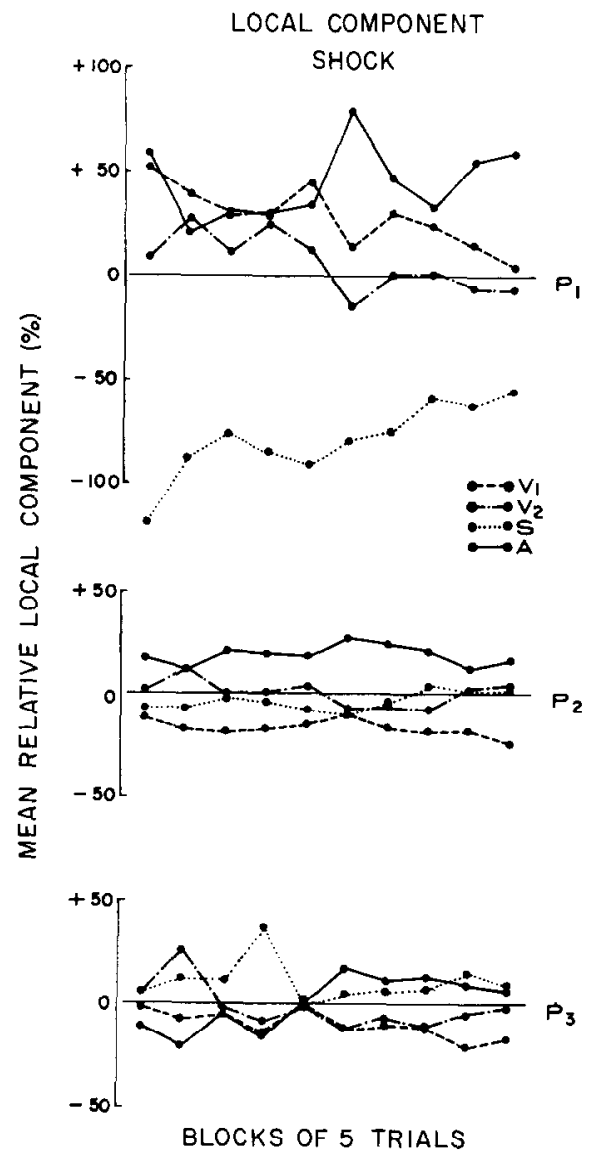

FIG. 9. Habituation of mean local component to shock. $N=12 . P_{1}$ : integration period one, period of stimulus. $\mathrm{P}_{2}$ : integration period two, first poststimulus period. $P_{3}$ : integration period three, second poststimulus period. $V_{1}$ : primary visual area. $\mathrm{V}_{z}$ : secondary visual area. $\mathrm{S}$ : somatic area. $\mathrm{A}$ : auditory area. For definition of mean relative local component, see text. 


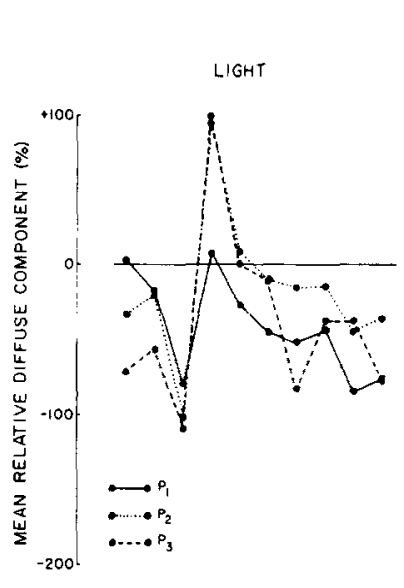

DIFFUSE COMPONENT

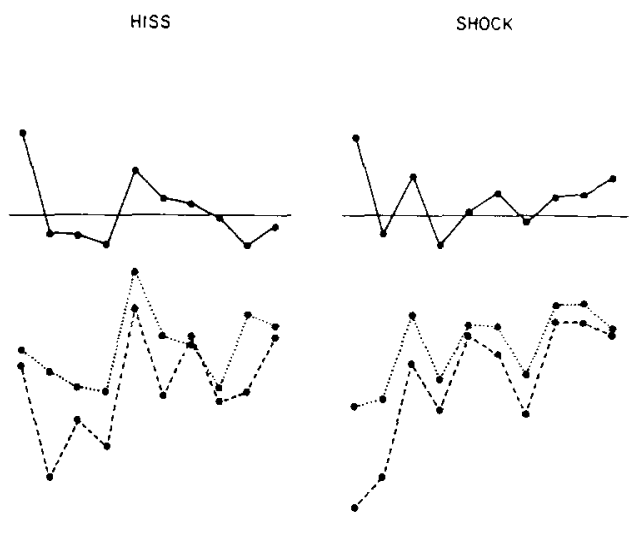

ELOCKS OF 5 TRIALS

Fic. 10. Habituation of mean diffuse component to light, hiss, and shock. Light: $N=8$. Hiss: $N=9$. Shock: $N=12, P_{1}$ : integration period one, period of stimulus. $P_{2}$ : integration period two, first poststimulus period. $P_{3}$ : integration period three, second poststimulus period. For definition of mean relative diffuse component, see text.

there is a suggestive trend toward increased negativity as trials proceed. This suggests that the habituation of the local component to light during $P_{1}$ (Fig. 7) is not the result of a general decrement in response size, but in a literal sense, the responses of the various areas become more nearly the same during a repetitive series.

\section{Discussion}

In evaluating these results it is of first importance to ask if the recorded signals could have arisen from non-neural sources of direct current. The use of calomel electrodes and chopper stabilized amplifiers ruled out such currents arising in the recording system. Immobilization minimized currents arising from somatic or ocular movements, and other investigators have reported the existence of $d-c$ responses in enucleated preparations (13, 27). Movement and dessication of the cortical probes was prevented by the use of a rigid head holder and by hermetic sealing of the junction between the probe and dura.

Second it may be asked if systemic physiologic changes, whether stimulus clicited or not, could contribute to the recorded signals. In the acute preparation this class of variables is difficult to hold under rigid control. Every attempt was made, however, to maintain systemic stability during 
the 1.5 to 2 hours of experimentation. Brain temperature and percentage of $\mathrm{CO}_{2}$ in respired air were maintained at stable values. The EKG and cortical SP were continuously monitored, and when instabilities developed, experimentation was interrupted until stability was regained. The eyes were atropinized to prevent changes in visual stimulus intensity due to changes in pupil diameter. That the responses are independent of stimulus elicited systemic reactions would seem to be proved by the cortical localization of the responses and their frequent close temporal correspondence with the onset and offset of stimulation (Fig. 2). In addition, several studies have described $d-c$ responses in cerveau isolé preparations in which the descending autonomic pathways are interrupted $(1,2,10)$.

It is both parsimonious and consistent, therefore, to interpret these sensory evoked $d-c$ responses as reflections of the combined activities of the specific and diffuse sensory projection systems. The local component is likely to reflect specific sensory activities, for as its name implies, it is localized according to the primary negative rule and it is temporally bound to the stimulus. Such responses have been reported by others as a result of electrical stimulation of thalamic relay nuclei $(2,6)$ as well as a result of receptor stimulation $(10,13)$.

The diffuse component may be presumed to be related to activity in the diffusely projecting systems. It is nonlocalized and it has a long latency, occurring principally as a poststimulation aftereffect. The stimuli which would be expected to elicit more intense diffuse cortical activation (shock and hiss, in that order) also elicit larger diffuse components. Responses of this type have been reported in the literature as a result of electrical stimulation of the diffusely projecting centers of the midbrain and thalamus $(1,9)$.

Both the local and diffuse components show a decremental tendency with repetition. As seen by the transient polarity reversal during the habituation of the diffuse component to light, however, the habituation curve is not necessarily a monotonically decreasing function. This may perhaps be explained by assuming that the habituation curve for $\mathrm{d}-\mathrm{c}$ responses reflects the summation of several unit activities, each habituating at slightly different rates. Phase relationships could cause one or another process to dominate at a particular moment.

The polarity reversal during habituation to light raises certain methodologic issues for experiments which must assume the long term stability of sensory evoked responses. Typical of such studies are those describing changes in electrographic indices due to stimulus pairing in a classical 
conditioning procedure. For instance, it has been reported that the d-c response evoked by a repetitive click stimulus can show a polarity reversal when the click is followed by shock to the radial nerve (23). On the basis of the present data, however, such polarity reversals may be possible independently of any pairing procedure.

Finally, it is appropriate to discuss the relevance of $d-c$ responses for the functioning of sensory mechanisms. It is extremely doubtful that surface potential changes of the magnitude reported here could significantly influence the functioning of neurons. Terzuolo and Bullock (25) measured the voltage gradient sufficient to modulate the frequency of a repetitively firing sensory neuron in the crayfish. The minimum value was reported to be about $10 \mathrm{mv} / \mathrm{cm}$. Changes of this magnitude in the vertically oriented cortical potential gradient may possibly occur during seizures and spreading depression, but not as a result of sensory stimulation.

Another line of argument is provided by recent studies relating direct cortical stimulation to sensory function. Libet et al. (18) found that, when applying electrical stimuli directly to the cortex, a current density of at least $5 \times 10^{-5} \mathrm{amp} / \mathrm{mm}^{2}$ was required to elicit conscious sensations in unanesthetized human patients, and based on a report by Doty (4), at least $5 \times 10^{-4} \mathrm{amp} / \mathrm{mm}^{2}$ was required to serve as a CS in a conditioning experiment using monkeys. In the former study, the value of $5 \times 10^{-5}$ was obtained by the use of a very large electrode (surface area $=78.5 \mathrm{~mm}^{2}$ ), and with smaller electrodes liminal current densities were about twenty times greater. Both studies used repetitive square-pulse stimulation, a procedure which would be expected to yield lower thresholds than $\mathrm{d}-\mathrm{c}$ polarization. Nevertheless, if currents of this small magnitude were to arise from the potentials typically recorded in the present study, it would imply a cortical specific resistance at least 1500 times smaller than the measured value of about 220 ohm-cm (22).

Among the smallest extracellular currents known to affect the functioning of mammalian cortical neurons are those reported by Rusinov (24) and Morrell (19). In the rabbit, surface positive polarization with $10^{-5}$ $\mathrm{amp} / \mathrm{mm}^{2}$ is effective in establishing a "dominant focus" of cortical excitability and may facilitate the response of single cortical neurons to sensory stimulation. Surface negative polarization of similar current density can produce a decrement in the performance of a partially learned conditioned avoidance responsc (20). In a study on rats, however, Kupfermann (16) found $10^{-5} \mathrm{amp} / \mathrm{mm}^{2}$ to be only marginally effective in disrupting visual discrimination learning. In addition, Kupferman measured the vertically 
oriented voltage gradient attendant to the artificially applied polarizing current. It was 20 to $40 \mathrm{mv} / \mathrm{mm}$ at 1 to $2 \mathrm{~mm}$ below the cortical surface. Gradients of this magnitude, which are barely effective in disturbing a complex discriminative behavior in the rat, are about 100 times greater than those which could be evoked by sensory stimulation.

It is likely, therefore, that sensory evoked extracellular d-c responses are a result, not a cause, of neural and perceptual activity in the cortex. Nevertheless, d-c responses, like EEG rhythms, can provide a useful indicator of the location and time course of active cortical foci. Such diagnostic tools are useful, perhaps even necessary, in the study of molar functions such as perception, attention and conditioning.

\section{References}

1. Arduini, A., M. Mancia, and K. Mechelse. 1957. Slow potential changes elicited in the cerebral cortex. Arch. Ital. Biol. 95: 127-138.

2. Brookhart, J., A. Arduini, M. Mancia, and B. Moruzzi. 1958. Thalamocortical relations as revealed by induced slow potential changes. $J$. Neurophysiol. 21: $499-525$.

3. Caspers, H. 1960. Changes in cortical d.c. potentials in the sleep-wakefulness cycle, pp. 237-259. In "Ciba Foundation Symposium on the Nature of Sleep." G. E. W. Wolstenholme and M. O'Connor [eds.]. Little, Brown, Boston, Massachusetts.

4. Doty, R. 1965. Conditioned reflexes elicited by electrical stimulation of the brain in macaques. J. Neurophysiol. 28: 622-640.

5. Gerard, R. 1936. Factors controlling brain potentials. Cold Spring Harbor Symp. Quant. Biol. 4: 292-304.

6. Goldring, S., and J. O'Leary. 1951. Summation of certain enduring sequelae of cortical activation in the rabbit. Electroencephalog. Clin. Neurophysiol. 3: 329-340.

7. Goldring, S., and J. O'Leary. 1954a. Correlation between steady transcortical potential and evoked responses. I. Electroencephalog. Clin. Neurophysiol. 6: 180-200.

8. Goldring, S., and J. O'Leary. 1954b. Correlation between steady transcortical potential and evoked responses. II. Electroencephalog. Clin. Neurophysiol. 6: 201-212.

9. Goldring, S., and J. O'Leary. 1957. Cortical d.c. changes incident to midline thalamic stimulation. Electroencephalog. Clin. Neurophysiol. 9: 577-584.

10. Gumnit, R. 1960. DC potential changes from auditory cortex of cat. J. Neurophysiol. 23: 667-675.

11. Gumnit, R. 1961. The distribution of direct current responses evoked by sounds in the auditory cortex of the cat. Electroencephalog. Clin. Neurophysiol. 13: 889-895.

12. KöhleR, W. and R. Held. 1949. The cortical correlate of pattern vision. Science 110: 414-419. 
13. Köhler, W., W. Neff, and J. Wegener. 1955. Currents of the auditory cortex in the cat. J. Cell. Comp. Physiol. 45 (Suppl. 1): 1-24.

14. Köhler, W., and D. O'Connell. 1957. Currents of the visual cortex of the cat. J. Cell. Comp. Physiol. 49 (Suppl. 2): 1-43.

15. Köhler, W., and J. Wegener. 1955. Currents of the human auditory cortex. J. Cell. Comp. Physiol. 45 (Suppl. 1): 25-54.

16. KupfermanN, I. 1965. Effects of cortical polarization on visual discriminations. Exptl. Neurol. 12: 179-189.

17. Lmet, B., and J. Kahn. 1946. Steady potentials and neuron activity in mammals. Federation Proc. 6: 152.

18. Libet, B., W. Alberts, E. Wright, L. Delattre, G. Levin, and B. Feinstein. 1964. Production of threshold levels of conscious sensation by electrical stimulation of human somatosensory cortex. J. Neurophysiol. 27: 546-578.

19. MorrelL, F. 1961. Effect of anodal polarization on the firing pattern of single cortical cells. Ann. N.Y. Acad. Sci. 92: 860-876.

20. Morrell, F., and P. Naitoh. 1962. Effect of cortical polarization on a conditioned avoidance response. Exptl. Neurol. 6: 507-523.

21. O'Leary, J, and S. Goldring. 1964. D-C potentials of the brain. Physiol. Rev. 44: 91-125.

22. Ranck, J. 1963. Specific impedance of rabbit cerebral cortex. Exptl. Neurol. 7: 144-152.

23. Rowland, V. 1960. Electrographic responses in sleeping conditioned animals, pp. 284-303. In "Ciba Foundation Symposium on the Nature of Sleep." G. E. W. Wolstenholme and M. O'Connor [eds.]. Little, Brown, Boston, Massachusetts.

24. Rusinov, V. 1953. An electrophysiological analysis of the connecting function in the cerebral cortex in the presence of a dominant region area. Communications XIX Internat. Physiol. Congr. Montreal: pp. 719-720.

25. Terzuolo, C., and T. Bullock. 1956. Measurement of imposed voltage gradient adequate to modulate neuronal firing. Proc. Natl. Acad. Sci. U.S. 42: 687-694.

26. Vanasupa, P., S. Goldring, J. O'Leary, and D. Winter. 1959. Steady potential changes during cortical activation. $J$. Neurophysiol. 22: 273-284.

27. Wurtz, R. 1965. Steady potential shifts during arousal and sleep in the cat. Electroencephalog. Clin. Neurophysiol. 18: 649-662. 